\title{
A New Triple-Well Resonant Tunneling Diode with Controllable Double-Negative Resistance
}

\author{
HIROSHI MIZUTA, TOMONORI TANOUE, AND SUSUMU TAKAHASHI, MEMBER, IEEE
}

\begin{abstract}
A newly structured triple-well resonant tunneling diode (RTD) is proposed as a triple-valued logic device. The superiority of this new RTD for triple-valued logic application is demonstrated with numerical simulation based on the transfer matrix method. This new RTD exhibits significant double-negative differential resistance, and the two current peak voltages are independently controlled with well thicknesses. Comparing the numerical calculation and experimental data, it is shown that agreement between the calculation and experiment on the two current peak voltages is excellent.
\end{abstract}

\section{INTRODUCTION}

$\mathrm{R}$ ECENTLY, resonant tunneling in multibarrier heterostructures [1], [2] has attracted considerable interest, and several resonant tunneling diodes (RTD) [3]-[10] and transistors [11]-[15] have been proposed as functional devices. One of the most hopeful applications of their negative differential resistance (NDR) is multiplevalued logic, which can greatly reduce the number of devices needed for one function [16]. For this application, multiple NDR where all peak currents are nearly equal is necessary. The simplest structure for an RTD with multiple NDR is a double-barrier heterostructure in which resonant levels are created in one quantum well. In this structure, however, the peak currents due to upper resonant levels are significantly larger than the peak current due to the lowest resonant level. This is caused by excess current whose origin is not yet determined. Thus, it is difficult to obtain the required multiple NDR by using the double-barrier structure. One certain method to avoid this difficulty is to arrange two double-barrier RTD's in parallel, where the NDR characteristics of the two diodes can be controlled with external bias [17]. This circuit, how- triple-well RTD, where the required double NDR can be realized, and the two current peak voltages are independently controllable with well thicknesses.

The method of our numerical calculations is shown in Section II. In Section III, the operation of the triple-well RTD as a triple-valued logic device is demonstrated. Comparison between the calculations and experiments is discussed in Section IV.

\section{FORMULATION}

In this section, we show the method of our calculation to analyze the resonant tunneling in multibarrier heterostructures. Our theoretical approach is based on the transfer matrix method [18], where the potential distribution of a resonant tunneling barrier (RTB) is approximated by a series of small steps as shown in Fig. 1. The wave function $\Psi_{i}(z)$ in the $i$ th section is given in a plane wave form as

$$
\Psi_{i}(z)=A_{i} \exp \left(i k_{i} z\right)+B_{i} \exp \left(-i k_{i} z\right)
$$

where $k_{i}$ is the complex wavenumber. Continuity of wave function and probability flux are required at all boundaries. When incident electrons come from the left side of RTB, the wave functions $\Psi_{L}$ and $\Psi_{R}$ on both sides of RTB are given by the following expressions:

$$
\begin{aligned}
& \Psi_{L}(z)=A_{L} \exp \left(i k_{L} z\right)+B_{L} \exp \left(-i k_{L} z\right), \quad z<z_{0} \\
& \Psi_{R}(z)=A_{R} \exp \left(i k_{R} z\right), \quad z>z_{N}
\end{aligned}
$$

and coefficients $A_{L}, B_{L}$, and $A_{R}$ are connected by transfer matrix $T_{i}$ as follows:

$$
\begin{aligned}
\left(\begin{array}{c}
A_{L} \\
B_{L}
\end{array}\right) & =T_{1} \cdot T_{2} \cdot T_{3}, \cdots, T_{N} \cdot T_{N+1}\left(\begin{array}{c}
A_{R} \\
0
\end{array}\right) \\
T_{i} & \equiv\left(\begin{array}{ll}
\alpha_{i}^{+} \exp \left(i\left(k_{i}-k_{i-1}\right) z_{i-1}\right) & \alpha_{i}^{-} \exp \left(-i\left(k_{i}+k_{i-1}\right) z_{i-1}\right) \\
\alpha_{i}^{-} \exp \left(i\left(k_{i}-k_{i-1}\right) z_{i-1}\right) & \alpha_{i}^{+} \exp \left(-i\left(k_{i}+k_{i-1}\right) z_{i-1}\right)
\end{array}\right) \\
\alpha_{i}^{ \pm} & \equiv \frac{1}{2}\left(1 \pm \frac{m_{i-1}^{*}}{m_{i}^{*}} \cdot \frac{k_{i}}{k_{i-1}}\right)
\end{aligned}
$$

ever, is more complex than that which uses a single diode. In this paper, we propose a new RTD structure, i.e., the

Manuscript received May 11, 1988; revised June 28, 1988.

The authors are with the Central Research Laboratory, Hitachi, Ltd., Kokubunji, Tokyo 185, Japan.

IEEE Log Number 8823793 where $m_{i}^{*}$ is the effective electron mass in the $i$ th section. The transmission probability $T$ through RTB is given as follows:

$$
T=\frac{m_{L}^{*}}{m_{R}^{*}} \cdot \frac{k_{R}}{k_{L}} \cdot \frac{\left|A_{R}\right|^{2}}{\left|A_{L}\right|^{2}}
$$




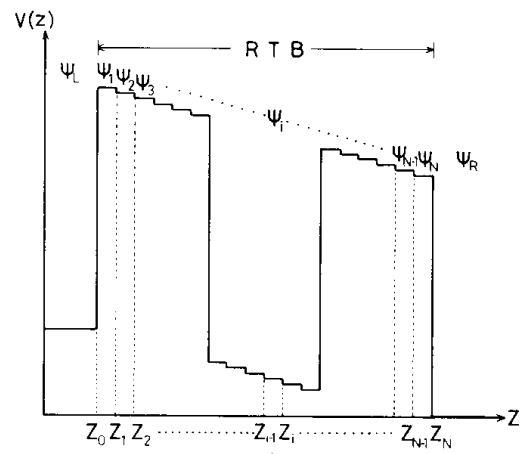

Fig. 1. A series of small steps used for the potential distribution under external bias

and tunnel current density $J_{T}$ is then calculated by the following formula:

$$
\begin{aligned}
J_{T}= & \frac{e k T}{2 \pi^{2} \hbar^{3}}\left[\int_{E_{C}^{L}}^{\infty} d E_{z} m_{R}^{*} T(E z)\right. \\
& \cdot \ln \left\{1+\exp \left(-\left(E_{z}-E_{F}^{L}\right) / k_{B} T\right)\right\} \\
& -\int_{E_{C}^{R}}^{\infty} d E_{z} m_{L}^{*} T(E z) \\
& \left.\cdot \ln \left\{1+\exp \left(-\left(E_{z}-E_{F}^{R}\right) / k_{B} T\right)\right\}\right]
\end{aligned}
$$

where $E_{C}^{L}$ and $E_{C}^{R}$ are conduction band edge energies, and $E_{F}^{L}$ and $E_{F}^{R}$ are the Fermi energies of electrons at both sides of RTB.

The effective electron mass in AlGaAs is expressed as follows:

$$
m^{*}=(0.067+0.083 x) m_{0}
$$

where $x$ is the Al mole fraction. For the electron effective mass in GaAs, the nonparabolicity of the $\Gamma$-band is approximately introduced by the following expression [19]:

$$
m^{*}(E)=0.067 m_{0}\left\{1-6 \alpha\left(E-E_{c}\right) / E_{g}\right\}
$$

where $E$ is the electron energy, $\alpha=-0.824$, and $E_{g}=$ $1.42 \mathrm{eV}$. The conduction band offset value is assumed to be 60 percent of the $\Gamma$ energy gap difference in the GaAs / AlGaAs heterostructure.

\section{Device Operation}

In this section, the operation of the triple-well RTD as a triple-valued logic device is demonstrated by using the theoretical approach described in Section II. Fig. 2 shows the band diagram of the newly proposed triple-well RTD, which consists of three quantum wells of undoped GaAs ( $W 1, W 2$, and $W 3$ ), four potential barriers (two undoped AlAs and two undoped $\mathrm{Al}_{0.26} \mathrm{Ga}_{0.74} \mathrm{As}$ ), and highly doped n-type $\operatorname{GaAs}\left(N_{D}=1.0 \times 10^{18} \mathrm{~cm}^{-3}\right)$ cathode and anode layers on both sides. The conduction band offset value between GaAs and AlAs is assumed to be $0.956 \mathrm{eV}$, and that between $\mathrm{GaAs}$ and $\mathrm{Al}_{0.26} \mathrm{Ga}_{0.74} \mathrm{As}$ to be $0.200 \mathrm{eV}$. The electron effective mass of $\mathrm{AlAs}$ is assumed to be

\begin{tabular}{|c|c|c|}
\hline \multicolumn{2}{|l|}{ Layer } & Widta $(\mathrm{nm})$ \\
\hline$i-A \mid A s$ & $\mathrm{La}_{1}$ & 1.0 \\
\hline 1-GaAs & L. 1 & 11.9 \\
\hline$-A 1 G a A s$ & $\mathrm{~L}_{32}$ & 3.0 \\
\hline - $-6 \mathrm{aAS}$ & $L * 2$ & 5.7 \\
\hline $1-11 \mathrm{GaAs}$ & $L_{B} 3$ & 3.0 \\
\hline $1-G a A s$ & $L_{1 / 3}$ & 6.9 \\
\hline $\mathrm{i}-\mathrm{A} I \mathrm{As}$ & $\mathrm{L}_{\mathrm{B} \text { d }}$ & 1.0 \\
\hline
\end{tabular}

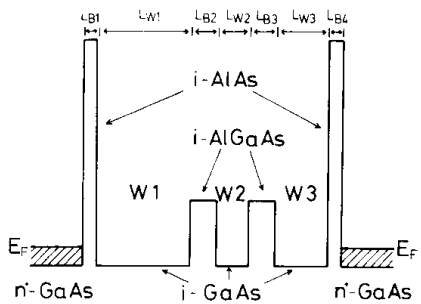

Fig. 2. Schematic energy-band diagram of triple-well resonant tunneling barrier and heavily doped electron regions.

TABLE I

Structure Parameters Used for Calculations

$0.15 m_{0}$, and that of $\mathrm{Al}_{0.26} \mathrm{Ga}_{0.74}$ As to be $0.0886 m_{0}$. Donors in the cathode and anode layers are assumed to be fully ionized, $N_{D}^{+}=N_{D}$, and electrons to be degenerate. Simulated results using structural parameters listed in $\mathrm{Ta}$ ble $I$ are shown in Fig. 3. The existent probability $\left|\Psi_{E}(z)\right|^{2}$ of electrons in RTB is shown in Fig. 3(a), and the energy dependence of the transmission probability $T(E)$ is shown in Fig. 3(b). In Fig. 3(a), $\left|\Psi_{E}(z)\right|^{2}$ is plotted for quasi-eigenenergies $E_{R}^{i}(i=1,2,3, \cdots$, numbered from the lowest energy) corresponding to the peaks in $T(E)$ [18]. Calculation is performed in the 0 to $0.22 \mathrm{eV}$ region, and there exists seven quasi-eigenstates. The three quasi-eigenstates $\Psi_{R}^{1}, \Psi_{R}^{2}$, and $\Psi_{R}^{3}$ are now utilized to give double NDR in $I-V$ characteristics as described in the following.

As shown in Fig. 3(a), $\left|\Psi_{R}^{1}(z)\right|^{2},\left|\Psi_{R}^{2}(z)\right|^{2}$, and $\left|\Psi_{R}^{3}(z)\right|^{2}$ are mainly located in the wells $W 1, W 3$, and $W 2$, respectively; consequently, their energies $E_{R}^{!}, E_{R}^{2}$, and $E_{R}^{3}$ are mostly determined by the well thickness $L_{W 1}$, $L_{W 3}$, and $L_{W 2}$. Here, the thickness of well $1, L_{W 1}$, is chosen so that $E_{R}^{1}<E_{F}$, where $E_{F}$ is the Fermi energy, and $L_{W 2}$ and $L_{W 3}$ so that $E_{F}<E_{R}^{2}<E_{R}^{3}$. Calculated values of the quasi-eigenenergies and the Fermi energy are listed in Table II, and it can be seen that the condition mentioned above is satisfied. It should be noted that the quasieigenstate $\Psi_{R}^{1}$ plays an important role in realizing the required two sharp current peaks. For this level operates as an injection level of electrons from the cathode layer, that is, the only electrons with energy close to $E_{R}^{1}$ are injected to $W 1$. The transmission probability of this level is now almost zero, but approaches 100 percent when quasi-eigenstate $\Psi_{R}^{2}$ or $\Psi_{R}^{3}$ just resonates with this injection level $\Psi_{R}^{1}$ under external bias. Therefore, the widths of the current peaks become very small, and valley currents can be decreased. It should be also noted that, by using the wave functions calculated above, the electron concentration in 


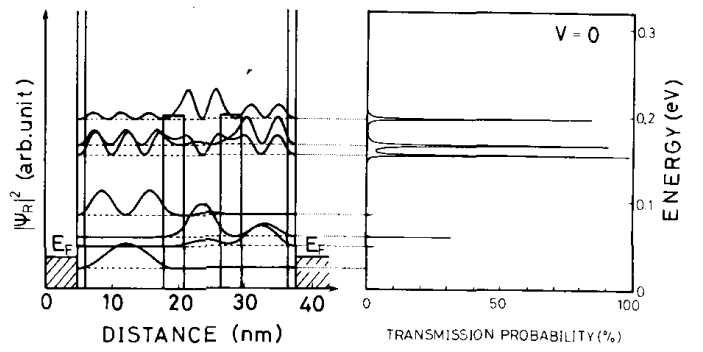

(a)

(b)

Fig. 3. (a) Existent probability $\left|\Psi_{E}(z)\right|^{2}$ of electrons in RTB for quasieigenenergies and (b) transmission probability $T$ versus energy in the 0 to $0.22 \mathrm{eV}$ region.

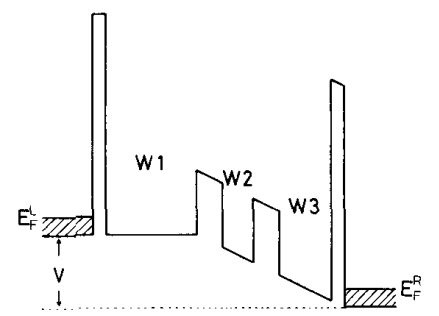

Fig. 4. Assumed energy-band diagram of triple-well RTD under external bias $V$.

TABLE II

Calculated Quasi-Eigenenergies $E_{R}^{1}-E_{R}^{7}$ AND the Fermi ENergy $E_{F}$

\begin{tabular}{c|c}
\hline \hline Quasi-eigenstate & Eigen-energy $(\mathrm{meV})$ \\
\hline$E_{\mathrm{R}}{ }^{2}$ & 23.5 \\
\hline$E_{\mathrm{R}^{2}}$ & 48.3 \\
\hline$E_{\mathrm{R}^{3}}$ & 59.5 \\
\hline$E_{\mathrm{R}}{ }^{4}$ & 84.5 \\
\hline$E_{\mathrm{R}}{ }^{5}$ & 153.6 \\
\hline$E_{\mathrm{R}^{6}}{ }^{6}$ & 105.2 \\
\hline$E_{\mathrm{R}^{7}}$ & 195.8 \\
\hline ermi energy $E_{\mathrm{F}}$ & 39.7 \\
\hline
\end{tabular}

the wells $W 1, W 2$, and $W 3$ can be estimated. The peak value of the electron concentration in $W 1\left(\sim 1 \times 10^{17}\right.$ $\left.\mathrm{cm}^{-3}\right)$ is much larger than that in $W 2\left(\sim 1 \times 10^{16} \mathrm{~cm}^{-3}\right)$ and that in $W 3\left(\sim 3 \times 10^{16} \mathrm{~cm}^{-3}\right)$ due to the relation among the quasi-eigenenergies and the Fermi energy $E_{R}^{1}<E_{F}<E_{R}^{2}<E_{R}^{3}$. Therefore, when external bias is applied to this RTD, a voltage drop is expected to occur almost in $W 2$ and $W 3$ because the electric field applied to $W 1$ is largely screened by the injected electrons. Thus, we assume that the band diagram under external bias can be approximated as shown in Fig. 4. A more exact band diagram can be determined by self-consistent calculation of the Schrödinger equation and Poisson's equation [20], but it would lead to longer computation, and we do not expect it to bring any interesting new device operation, only better accuracy.

Calculated $I-V$ characteristics where double NDR appears are shown in Fig. 5. In Fig. 6(a)-(c), $\left|\Psi_{E}(z)\right|^{2}$ and $T(E)$ are plotted for three typical points of $I-V$ charac-

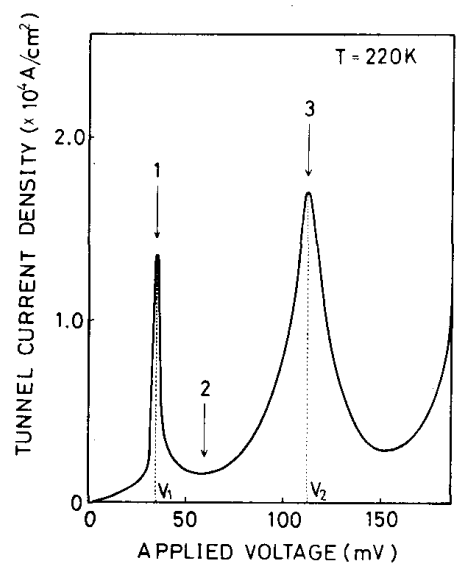

Fig. 5. Calculated current-voltage characteristics of triple-well RTD Three typical points are pointed out by arrows: arrow 1 for the first current peak, 2 for the current valley, and 3 for the second current peak.

(a)

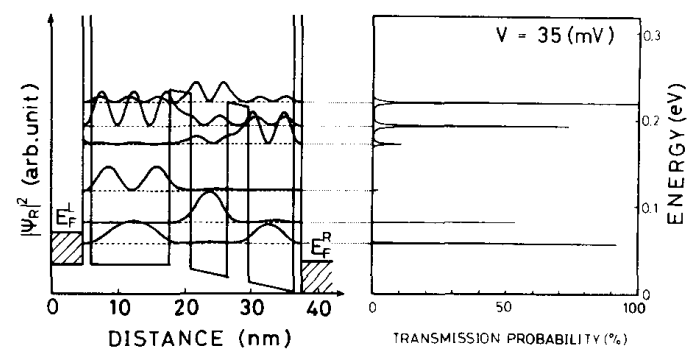

(b)

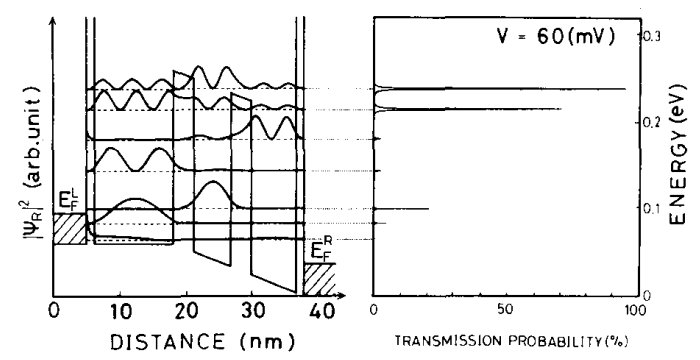

(c)

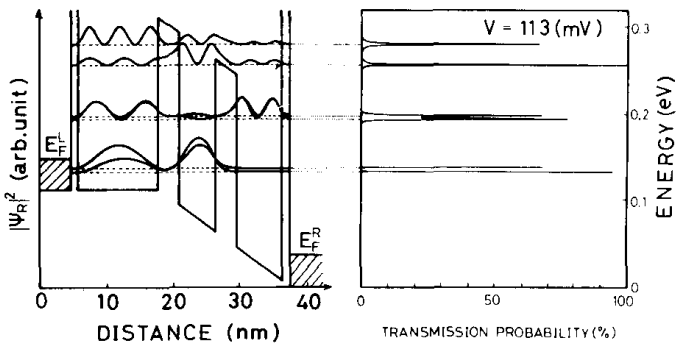

Fig. 6. Plots of $\left|\Psi_{E}(z)\right|^{2}$ and $T(E)$ for three typical points of the $I-V$ characteristics shown in Fig. 5: (a) first resonant state (arrow 1), (b) offresonant state (arrow 2), and (c) second resonant state (arrow 3).

teristics: first resonant state, off-resonant state, and second resonant state. As shown in these figures, the first current peak in the $I-V$ characteristics originates from the resonance of $\Psi_{R}^{2}$ with $\Psi_{R}^{1}$, and the second from that of 


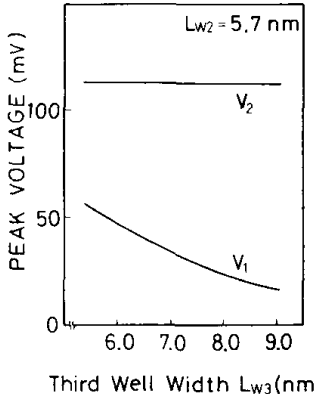

(a)

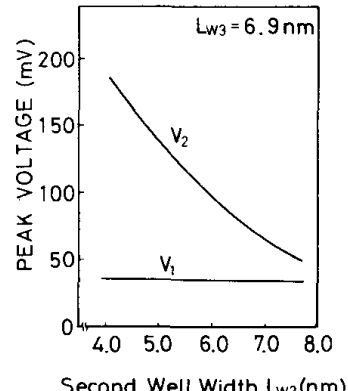

(b)
Fig. 7. Peak voltages versus well widths: (a) $L_{W 3}$ dependence of the peak voltages $V_{1}$ and $V_{2}$ and (b) $L_{W 2}$ dependence of peak voltages.

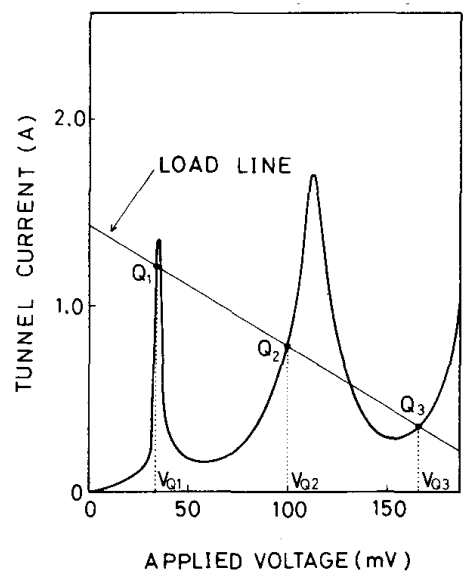

Fig. 8. The current-voltage characteristics and the load line. Three stable points $Q_{1}, Q_{2}$, and $Q_{3}$ are located at the voltages $V_{Q 1}, V_{Q 2}$, and $V_{Q 3}$.

$\Psi_{R}^{3}$ with $\Psi_{R}^{1}$. Therefore, two peak voltages $V_{1}$ and $V_{2}$ (see Fig. 5) are determined by the energy separation between $E_{R}^{1}$ and $E_{R}^{2}$ and that between $E_{R}^{1}$ and $E_{R}^{3}$, respectively. Namely, two peak voltages can be independently controlled with the well thicknesses $L_{W 3}$ and $L_{W 2}$. This controllability is shown in Fig. 7: (a) the $L_{W 3}$ dependence of the peak voltages $V_{1}$ and $V_{2}$ and (b) the $L_{W 2}$ dependence of $V_{1}$ and $V_{2}$. These figures clearly show that the first peak voltage can be controlled by the thickness of $W 3$ without changing the second peak voltage, and similarly the second peak voltage by that of $W 2$. Such an indepenedent controllability of two current peak voltages is a special feature of the triple-well RTD.

When the triple-well RTD of $100 \times 100 \mu \mathrm{m}^{2}$ is operated with a load resistance of $0.15 \Omega$ and an applied voltage of $220 \mathrm{mV}$, the calculated three stable points $Q_{1}, Q_{2}$, and $Q_{3}$ are located at 34,100 , and $166 \mathrm{mV}$ as shown in Fig. 8. Structural parameters are chosen so that voltage intervals between them are equal to $66 \mathrm{mV}$, and this value can be also varied with the well thicknesses $L_{W 3}$ and $L_{W 2}$, as explained above. Therefore, the ideal $I-V$ characteristics for triple-valued logic can be designed using this triple-well RTD.

\section{Discussion}

\section{A. Peak Voltages $V_{1}$ and $V_{2}$}

Based on the numerical calculation described above, a triple-well RTD has been fabricated [21]. Fig. 9 shows a cross section of the triple-well RTD, where a 10-nm-thick undoped GaAs offset layer is introduced under RTB to avoid diffusion of silicon ions into the triple-well structure from the $\mathrm{n}^{+}-\mathrm{GaAs}$ anode layer. As shown in Fig. 10, the fabricated RTD exhibits a significant double NDR that is ideal for triple-valued logic. It was measured at a temperature of $220 \mathrm{~K}$ where two peak currents were nearly equal; the current density of the second peak depends on temperature as discussed in the next section. We have fabricated several RTD's ranging from $4 \times 4$ to $100 \times$ $100 \mu \mathrm{m}^{2}$ in size and observed changes of the two peak voltages. As shown in Fig. 11, both of the two peak voltages are found to depend linearly on the diode current through a resistance of approximately $4 \Omega$. This resistance is thought to be induced by the external measurement system and is in series with the diode. Intrinsic values of the peak voltages are obtained at the intersections of $V_{1}$ and $V_{2}$ with the vertical axis $\left(I_{P}=0\right)$. To compare experimental with theoretical values of these peak voltages, it is necessary to subtract the voltage drop in the offset layer: the corrected values $V_{1}^{*}$ and $V_{2}^{*}$ are obtained from measured values as follows:

$$
\begin{aligned}
& V_{i}^{*}=\frac{L_{E}-L_{\mathrm{OFFSET}}}{L_{E}} V_{i}, \quad i=1,2 \\
& L_{E}=L_{B 2}+L_{W 2}+L_{B 3}+L_{W 3}+L_{B 4}+L_{\mathrm{OFFSET}}
\end{aligned}
$$

where $L_{\text {OFFSET }}$ is the thickness of the offset layer. Theoretical and experimental values after correction are shown in Table III. This excellent agreement between the calculated and experimental values signifies the propriety of the simplified assumption for the energy band diagram. Thus, it is shown that the two current peak voltages $V_{1}$ and $V_{2}$ can be quantitatively estimated with our numerical calculation.

\section{B. Peak Current Density $J_{P}^{1}$ and $J_{P}^{2}$}

As shown in Section IV-A, the two observed peak voltages $V_{1}$ and $V_{2}$ are in excellent agreement with calculated values. Up to now, however, comparison between calculated and experimental peak currents has not been made. In this section, the comparison is made between the calculated and experimental peak current densities, and improvement in the model of the calculation is discussed.

Fig. 12 shows temperature dependences of the calculated and experimental peak current densities $J_{P}^{1}$ and $J_{P}^{2}$. It can be seen that the first peak current density decreases and the second increases with a rise in temperature. This temperature dependence is theoretically explained as follows.

1) As temperature rises, the number of electrons at higher energies increases and that at the resonant en- 


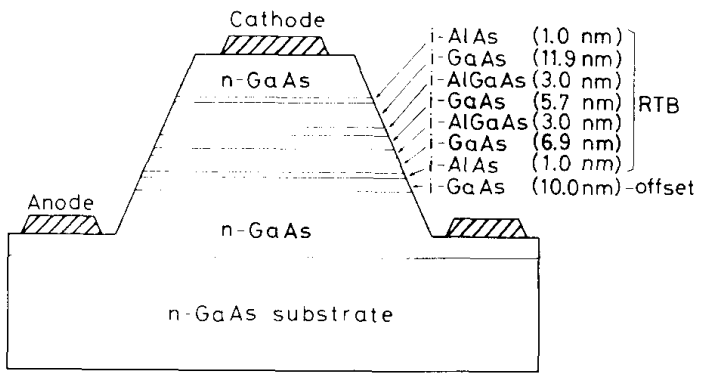

Fig. 9. Schematic cross section and layer thicknesses of a fabricated triplewell RTD.

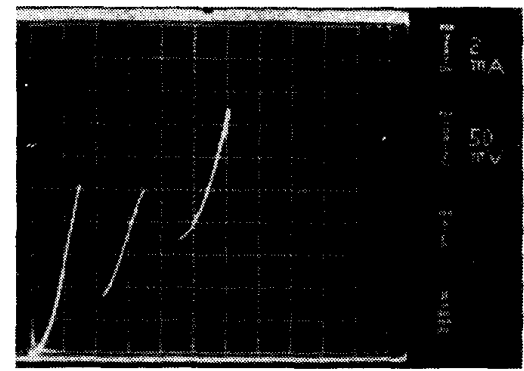

Fig. 10. Typical $I-V$ characteristics $(220 \mathrm{~K})$ of a triple-well RTD.

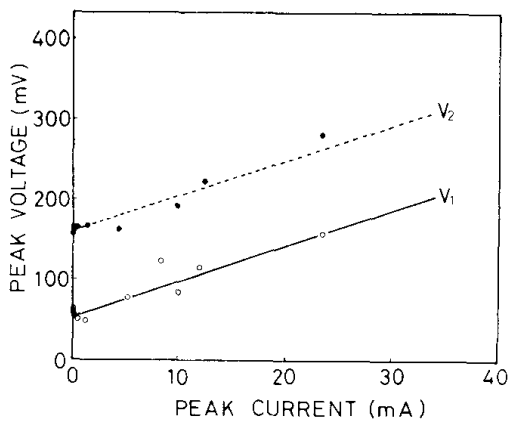

Fig. 11. Dependence of two peak voltages $V_{1}$ and $V_{2}$ on peak currents.

TABLE III

Calculated and Experimental Peak Voltages $V_{1}$ and $V_{2}$

\begin{tabular}{c|c|c}
\hline & $V_{1}(\mathrm{mV})$ & $V_{2}(\mathrm{mV})$ \\
\hline Calculated & 35 & 113 \\
\hline Experimental & 33 & 106 \\
\hline
\end{tabular}

ergy decreases. Thus, the first peak current decreases.

2) At the second resonance (resonance of $\Psi_{R}^{3}$ with $\Psi_{R}^{1}$ ), another resonance ( resonance of $\Psi_{R}^{5}$ with $\Psi_{R}^{4}$ ) also occurs as shown in Fig. 6(c). The number of electrons that have enough energy to pass this higher resonant level increases at high temperatures; therefore, the second peak current increases.

The experimental peak current densities show qualitatively the same temperature dependence as the calculated ones. Their absolut z values, however, are more than one order of magnitude smaller than the calculated values. These facts signify that our understanding of the resonant

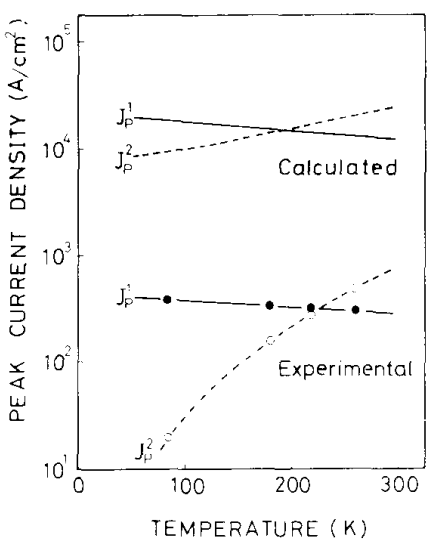

Fig. 12. Calculated and experimental peak currents versus temperature.

TABLE IV

Calculated and Experimfntal Half-Widths $\Gamma_{\text {, And }} \Gamma$, of Two Current Peaks

\begin{tabular}{l|c|c}
\hline \hline & $\Gamma_{1}(\mathrm{mV})$ & $\Gamma_{2}(\mathrm{~m} V)$ \\
\hline Calculated & 7 & 21 \\
\hline Experimental & 25 & 40 \\
\hline
\end{tabular}

mechanism explained in Section III is fundamentally correct, but the number of electrons at the resonant energies is overestimated in our calculation. As a reference, comparison between calculated and experimental peak current densities was made in double-barrier RTD with two AIAs barriers, and the disagreement between them was less than a factor 3. Thus, the discrepancy in the peak current densities of the triple-well RTD is peculiar to this structure. The most significant difference between the double-barrier RTD and our triple-well RTD is the existence of well 1 , i.e., the injection level $\Psi_{R}^{1}$. As explained in Section III, this level acts as an injection level, and the only electrons in the cathode layer with energy nearly equal to $E_{R}^{1}$ can go into well 1 . Tunnel current flows only when the quasi-eigenstate $\Psi_{R}^{2}$ or $\Psi_{R}^{3}$ just resonants with this level. Such resonant tunneling mechanisms bring sharp current peaks and high peak current densities. However, the energy uniformity of the electrons injected into well 1 can be lost by the scattering processes such as LO-phonon, and $\Gamma \rightarrow X$ intervalley scattering, which are ignored in our calculation. These scattering processes broaden the width of the injection level in energy space and cause the energy relaxation of the electrons in well 1 , resulting in the decrease of the peak currents. The observed halfwidths (full width at half-maximum) of the two current peaks and the calculated ones are listed in Table IV. As shown in this table, the observed values are two or three times larger than the calculated ones. This broadening of the current peaks implies the existence of the scattering effects described above.

A new theoretical approach taking into account the scattering mechanisms explained above is currently being developed, and preliminary calculations indicate that the difference between the calculated and experimental peak current densities seems to be reduced. Details of this calculation will be reported elsewhere. 


\section{CONCLUSION}

A newly structured triple-well RTD has been proposed and theoretically analyzed for a triple-valued logic device. The superiority of the triple-well RTD for triplevalued logic application is demonstrated with numerical simulation based on the transfer matrix method. In this structure, there exist three quasi-eigenstates $\Psi_{R}^{1}, \Psi_{R}^{2}$, and $\Psi_{R}^{3}$, corresponding to the ground states of triple wells $W 1$, $W 3$, and $W 2$. When external bias is applied, the resonance of $\Psi_{R}^{2}$ with $\Psi_{R}^{1}$ causes the first current peak, and that of $\Psi_{R}^{3}$ with $\Psi_{R}^{1}$ causes the second. Thus, this new RTD exhibits significant double-negative resistance, and the two current peak voltages are independently controlled with the thicknesses of wells $W 3$ and $W 2$. The controllability of two current peak voltages is characteristic of the triple-well RTD. Comparing the numerical calculation and experimental data, the calculated two current peak voltages are in excellent agreement with experimental ones.

\section{ACKNOWLEDGMENT}

The authors wish to thank M. Kobayashi and Dr. H. Yanazawa and his staff for device fabrication, C. Kusano for his help in low-temperature measurements, and Dr. H. Matsumura for his encouragement.

\section{REFERENCES}

[1] R. Tsu and L. Esaki, "Tunneling in a finite superlattice," Appl. Phys. Lett., vol. 22, no. 11, pp. 562-564, 1973.

[2] F. Capasso, K. Mohammed, and A. Y. Cho, "Resonant tunneling through double barriers, perpendicular quantum transport phenomena in superlattices, and their device applications," IEEE J. Quantum Electron., vol. QE-22, no. 9, pp. 1853-1869, 1986.

[3] S. Muto, T. Inata, H. Ohnishi, N. Yokoyama, and S. Hiyamizu, "Effect of silicon doping profile on $I-V$ characteristics of an $\mathrm{Al}-$ GaAs/GaAs resonant tunneling barrier structure grown by MBE," Japan. J. Appl. Phys., vol. 25, no. 7, pp. L577-L599, 1986.

[4] T. Inata, S. Muto, Y. Nakata, T. Fujii, H. Ohnishi, and S. Hiyamizu, "Excellent negative differential resistance of InAlAs /InGaAs resonant tunneling barrier structure grown by MBE," Japan. J. Appl. Phys., vol. 25, no. 12, pp. L983-L985, 1986.

[5] G. Sollner, W. D. Goodhue, P. E. Tannenwald, C. D. Parker, and D. D. Peck, "Resonant tunneling through quantum wells at frequencies up to $2.5 \mathrm{GHz}$," Appl. Phys. Lett., vol. 43, no. 6, pp. 588-590, 1983 .

[6] M. Tsuchiya, H. Sakaki, and J. Yoshino, "Room temperature observation of differential negative resistance in an AlAs/GaAs/AlAs resonant tunneling diode," Japan. J. Appl. Phys., vol. 24, no. 6, pp. L466-L468, 1985.

[7] M. Tsuchiya and H. Sakaki, "Dependence of resonant tunneling current on well widths in AlAs / GaAs/AlAs double barrier diode structures," Appl. Phys. Lett., vol. 49, no. 2, pp. 88-90, 1986.

[8] - "Precise control of resonant tunneling current in AlAs/GaAs/AlAs double barrier diodes with atomically-controlled barrier widths," Japan. J. Appl. Phys., vol. 25, no. 3, pp. L185L187, 1986.

[9] T. Nakagawa, H. Inamoto, T. Kojima, and K. Ohta, "Observation of resonant tunneling in AlGaAs/GaAs triple barrier diodes," Appl. Phys. Lett., vol. 49, pp. 73-75, 1986.

[10] S. Sen, F. Cappaso, A. C. Gossard, R. A. Spah, A. L. Hutchinson, and S. N. G. Chu, "Observation of resonant tunneling through a compositionally graded parabolic quantum well," Appl. Phys. Lett., vol. 51 , no. 18 , pp. $1428-1430,1987$

[11] N. Yokoyama, K. Imamura, S. Muta, S. Hiyamizu, and H. Nishi, "A new functional, resonant-tunneling hot electron transistor (RHET)," Japan. J. Appl. Phys., vol. 24, no. 11, pp. L853-L854, 1985.

[12] F. Capasso, S. Sen, A. C. Gossard, A. L. Hutchinson, and J. H. English, "Quantum well resonant tunneling bipolar transistors oper-

ating at room temperature," IEEE Electron Device Lett., vol. EDL7, pp. 573-576, 1986

[13] F. Capasso, S. Sen, and A. Y. Cho, "Negative transconductance resonant tunneling field-effect transistor," Appl. Phys. Lett., vol. 51, no. 7 , pp. $1768-1772,1987$.

[14] A. R. Bonnefoi, T. C. Mcgill, and R. D. Burnham, "Resonant tunneling transistors with controllable negative differential resistances," IEEE Electron Device Lett., vol. EDL-6, no. 12, pp. 636-638, 1985.

[15] S. Sen, F. Capasso, F. Beltram, and A. Y. Cho, "The resonant-tunneling field effect transistor: A new negative transconductance device," IEEE Trans. Electron Devices, vol. ED-34, no. 8, pp. 1768$1773,1987$.

[16] C. Rine, Ed., Computer Science and Multiple-Valued Logic. Amsterdam: North-Holland, 1984, p. 415.

[17] F. Capasso, S. Sen, A. Y. Cho, and D. SIvico, "Resonant tunneling devices with multiple negative differential resistance and demonstration of three-state memory cell for multiple-valued logic applications," IEEE Electron Device Lett., vol. EDL-8, pp. 297-299, 1987.

[17] A. Harwit and J. S. Harris, Jr., "Calculated quasi-eigenstates and quasi-eigenenergies of quantum well superlattices in an applied electric field," J. Appl. Phys., vol. 60, no. 9, pp. 3211-3213, 1986.

[19] J. S. Blakemore, "Semiconducting and other major properties of gallium arsenide," J. Appl. Phys., vol. 53, no. 19, p. R160, 1982.

[20] H. Ohnishi, T. Inata, S. Muto, N. Yokoyama, and A. Shibatomi, "Self-consistent analysis of resonant tunneling current," Appl Phys. Lett., vol. 49, no. 19, pp. 1248-1250, 1986.

[21] T. Tanoue, H. Mizuta, and S. Takahashi, IEEE Electron Device Lett., submitted for publication.

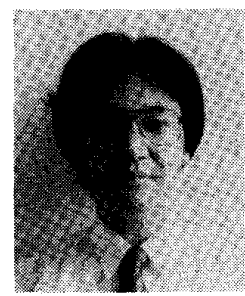

Hiroshi Mizuta was born in Kochi, Japan, in 1961. He received the B.S. and M.S. degrees in physics from Osaka University, Osaka, Japan, in 1983 and 1985 , respectively.

Since 1985, he has been working for the Central Research Laboratory, Hitachi, Ltd., Tokyo, on compound heterojunction devices. His current research interests are on device physics, device modeling, simulation technology, and new functional devices.

Mr. Mizuta is a member of the Physical Society of Japan and the Japan Society of Applied Physics.

*

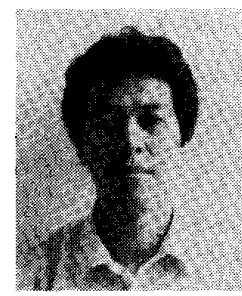

Tomonori Tanoue was born in Tokyo, Japan, in 1957. He received the engineering degrees of bachelor, master, and doctor from the University of Tokyo, Tokyo, Japan, in 1980, 1982, and 1986, respectively.

Since he joined the Central Research Laboratory, Hitachi, Ltd., in 1987, his main concern has been the growth and fabrication of heterojunction devices such as MQW lasers, HBT's, heterojunction FET's, and resonant tunneling devices. He is currently working for new functional devices using resonant tunneling barriers.

Dr. Tanoue is a member of the Japan Society of Applied Physics and the Institute of Electronics, Information and Communication Engineers of Japan.

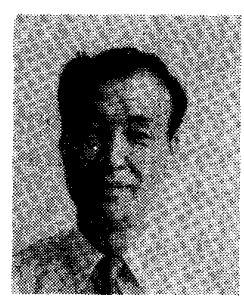

Susumu Takahashi (M'82) was born in Iwate, Japan, in 1944. He received the B.Sc. degree in physics from the University of Hirosaki, Hirosaki, Japan, in 1967 and the Ph.D. degree in electrical engineering from the University of Tokyo, Tokyo, Japan, in 1979.

Since 1967, he has been working for the Central Research Laboratory Hitachi, Ltd., Tokyo, Japan, on low-noise GaAs MESFET's, silicon devices, and GaAs LSI's. His current research interests are compound hetero devices and technol-

ogy.

Dr. Takahashi is a member of the Japan Society of Applied Physics and the Institute of Electronics, Information and Communication Engineers of Japan. 